\title{
La ética en el proceso militar para la toma de decisiones: en el interior del planeamiento operacional $^{1}$
}

\author{
Hamilton Osiris Jiménez Ortiz \\ Escuela Superior de Guerra "General Rafael Reyes Prieto" \\ Carlos Augusto Paez Murillo 3 \\ Escuela Militar de Cadetes "General José María Córdova” \\ Robinson Cadena Bareño ${ }^{4}$ \\ Universitat Jaume I
}

1 Este capítulo presenta los resultados colaborativos de dos proyectos de investigación: (1) "Ética militar en entornos complejos de seguridad y defensa: lecturas y aportes desde la experiencia de las Fuerzas Militares de Colombia”, del grupo de investigación Masa Crítica de la Escuela Superior de Guerra "General Rafael Reyes Prieto", Colombia, categorizado en B por Minciencias y con código de registro COL0123247, y (2) "Mujeres de arma, seguridad y defensa nacional. Un análisis desde sus percepciones", del grupo de investigación en Ciencias Militares, de la Escuela Militar de Cadetes "General José María Córdova", Colombia, categorizado en B por Minciencias y con código de registro COL0082556. Los puntos de vista pertenecen a los autores y no reflejan necesariamente los de las instituciones participantes.

2 Magíster en Estudios de Seguridad Internacional de la Universidad de Defensa Nacional, Washington. Magíster en Derechos Humanos, Derecho Internacional Humanitario y Derecho Operacional de la Universidad de Nebrija. Especialista en Seguridad y Defensa Nacionales, Escuela Superior de Guerra "General Rafael Reyes Prieto". Docente de la Escuela Superior de Guerra "General Rafael Reyes Prieto", Colombia, y asesor de defensa e inteligencia estratégica para el Comando General de las Fuerzas Militares, Colombia. OrciD: https://orcid.org/0000-0002-1868-8670 - Contacto: hamilton.jimenez@esdegue.edu.co

$3 \mathrm{PhD}$ en Ciencias en Ingeniería de Sistemas del Instituto Politécnico Nacional de México. Magíster en Ciencias en Ingeniería de Sistemas del Instituto Politécnico Nacional de México. Especialista en Administración de Recursos Militares del Centro de Educación Militar. Docente de la Escuela Militar de Cadetes "General José María Córdova", Colombia. Orcid: https://orcid.org/0000-0002-5221-8437 Contacto: carlos.paez@esmic.edu.co

$4 \mathrm{PhD}$ (c) y magíster en Estudios Internacionales de Paz, Conflictos y Desarrollo de la Universitat Jaume I, España. Magíster en Seguridad y Defensa Nacionales de la Escuela Superior de Guerra "General Rafael Reyes Prieto". Master of Policing, Intelligence and Counterterrorism with master of International Security Studies de la Universidad Macquarie, Australia. Investigador PDI Universitat Jaume I, España. OrCID: https://orcid.org/0000-0002-4325-4380 - Contacto: rcadena@uji.es 


\section{Resumen}

El proceso militar para la toma de decisiones (PMTD) es el planeamiento que las planas y estados mayores desarrollan para que las unidades militares ejecuten posteriormente operaciones militares en el amplio espectro de su misionalidad, tanto en tiempos de paz como de guerra. Ante tal importancia, la ética militar ha sido un determinante fundamental desde el punto de vista práctico, no obstante, dentro de la concepción teórica de las operaciones o planeamiento se deja por sentada su presencia y, en consecuencia, se resta el interés que esta requiere. Por tanto, el objetivo del presente documento es describir un marco ético de aplicación en el PMTD a partir de una investigación documental, en la cual se encontró que el modelo del triángulo ético y la medición de incertidumbre para anticipar dilemas éticos presentan un marco ético inicial y apropiado para complementar el PMtd.

Palabras clave: conflicto; ética; operaciones militares; planeamiento; proceso militar para la toma de decisiones; profesión militar.

\section{Introducción}

Los escenarios de las relaciones internacionales, la seguridad y defensa son ampliamente complejos hoy en día y presentan grandes desafíos para los Estados, sobre todo para aquellos que poseen el sistema de gobierno democrático (Jordán, 2016). Como herramienta del Estado se encuentran las Fuerzas Militares, que conducen sus operaciones dentro de los mencionados escenarios y por lo cual sus acciones cada vez más enfrentan mayores retos que exigen una mejor preparación (Muñoz, 2013). Debido a la globalización y a la quinta revolución de las comunicaciones, los organismos militares se enfrentan al cuestionamiento continuo de sus acciones, ya que narrativas antidemocráticas han proliferado en el mundo occidental como parte del efecto de polarización profundo que ocurre actualmente y que ha podido ser observado de forma clara en países como Estados Unidos y Colombia (Rayón, 2018). Estos cuestionamientos procuran despojar de legitimidad las diferentes acciones que ejecutan las Fuerzas Militares en ambientes nacionales o internacionales, al tratar de manera constante de atacar los marcos legales, jurídicos y/o constitucionales por los que normalmente se rigen. 
La profesión militar, entonces, enfrenta uno de sus momentos más cuestionados al ser atacada en el núcleo de su propósito y al ponerse bajo juicio lo imprescindible de su existencia (Von Bredow, 2011). Pero los riesgos a la institución militar no solo provienen de actores antidemocráticos y antimilitaristas, sino que también se han sistematizado dentro de los procesos de control y auditoría en el interior del propio sistema democrático. Precisamente, la condición democrática del gobierno y la característica de autoridad civil sobre la defensa exigen la verificación continua de las acciones del personal militar respecto a su misión constitucional (Travis, 2019). Teniendo claro que las operaciones militares se encuentran bajo la supervisión de organismos de control democráticos y de actores que pretenden poner en duda la legitimidad de las Fuerzas para afectar al Estado, se puede determinar que dicho escenario se examina en el plano de la ética. Es en ella en donde se han presentado los mayores cuestionamientos a las acciones militares y desde donde se argumentan posiciones de ilegitimidad, ilegalidad, violaciones al derecho internacional e incluso crímenes de lesa humanidad.

Se conforma entonces un imperativo en el comportamiento de la profesión militar, la cual debe basarse en un marco ético coherente y adecuado para el ejercicio legítimo del uso de la fuerza (Cook, 2000). Las fuerzas militares contemporáneas han logrado grandes avances en el mejoramiento de sus referentes éticos y se han enmarcado en los lineamientos de los Derechos Humanos, con lo cual su profesionalización ha logrado cohesionar la autoridad y los poderes del Estado (Aristizábal et al., 2020). Sin embargo, los cuestionamientos éticos persisten hasta hoy, ya que la característica única de la profesión militar, como la posesión de las armas del Estado y el ejercicio legítimo de la violencia, generará contradicciones en las que se tratará de argumentar acciones incorrectas por las unidades en el terreno.

Los organismos militares actúan a través de la operacionalidad (Valdés, 2020). El poder que estos aplican se pone en práctica mediante operaciones militares en un terreno, territorio, ambiente o superficie que posee un entorno con el cual interrelacionan. La generalidad operacional en el contexto de doctrina y de formación personal ha sido ampliamente transversalizada con elementos claros de conceptualización hacia la ética, de tal modo que la 
mención de ella está incluida en manuales fundamentales y de liderazgo en las Fuerzas Militares actuales. Estas operaciones, antes de ser llevadas a la práctica, son concebidas a través de un proceso militar para la toma de decisiones (РмTD), que se compone de siete pasos definidos, en donde se construye el enfoque para la ejecución de la acción militar (Fotion \& Elfstrom, 1986), que es desarrollado por una plana o un estado mayor, ya sea orgánico o superior (Alvinius et al., 2018). Por ende, el vacío ético consiste en que la doctrina para los procedimientos o procesos de planeamiento militar no contiene consideraciones éticas relacionadas con el proceso en sí mismo, sino que el parámetro de lo correcto se encuentra asociado puramente al juicioso seguimiento y realización de las instrucciones para cada uno de los siete pasos del proceso (Kem, 2006). Es decir que el planeador militar que siga acuciosamente el PMTD aparentemente no cometería ninguna falta ética en el proceso.

Los miembros de las Fuerzas Militares de Colombia, en su experiencia durante el conflicto armado interno, han observado que es necesario complementar los procesos de planeamiento con consideraciones éticas específicas y diseñadas para lograr mejor visibilidad. Esta demanda motiva la pregunta de investigación: ¿Cuál es el marco ético que debe ser aplicado en el proceso militar para la toma de decisiones con el fin de garantizar una legítima conducción de operaciones militares? Por lo anterior, el objetivo del presente documento es describir un marco ético de aplicación en el proceso militar para la toma de decisiones con el fin de garantizar la legitimidad de la conducción de operaciones militares.

\section{Marco teórico}

\section{Naturaleza de la profesión militar}

La profesión militar es la actividad en la cual los integrantes de las fuerzas militares legítimas de un país actúan con el fin de defender los intereses nacionales y proteger su territorio y su población, generalmente bajo una autoridad civil y enmarcados en una constitución nacional (US Army, 2019b). Después de la Segunda Guerra Mundial, las fuerzas militares tuvieron que hacerse cada vez más partícipes en los asuntos de seguridad debido a la explosión de movi- 
mientos insurgentes a nivel mundial, causados principalmente por los procesos de descolonización y las luchas socialistas que surgieron ante inconformismos sociales y políticos, sobre todo en países de América Latina.

Bien sea por asuntos de seguridad o de defensa, la profesión militar se ha encontrado totalmente relacionada al fenómeno del conflicto armado o de la guerra como herramienta del Estado con el fin de mantener el sistema de gobierno establecido (Park, 2018). Por lo tanto, la acción pública de los militares se enfrenta sistemáticamente a un continuo ciclo de toma de decisiones, del cual dependen las vidas de las propias tropas, del adversario (cuando lo hay) y la propia integridad de terceros, ya sea personal civil y/u organizaciones de diferente índole. Es así como el grado de responsabilidad en la profesión militar es mayúsculo en este aspecto debido a la proximidad e involucramiento, ya sea voluntario o involuntario, de estos actores en la conducción de las operaciones militares.

\section{Contexto histórico de ética en lo militar}

La ética se define como el pensamiento moral en el que se decide el uso del bien o del mal en el comportamiento humano y que ha estado presente en el ser humano desde la formación del concepto de familia y la correspondiente conformación de grupos sociales (Polo, 2016). El razonamiento ético posee una tensión constante entre la idealidad y la realidad en la actuación humana, dentro de la cual se plantea la acción como una representación de lo establecido y generalmente aceptado como correcto (González, 1999).

La sociedad y el Estado moderno han sostenido discusiones morales a lo largo de su progreso y en cada avance han cuestionado acciones del pasado con el fin de reconstruir nuevos códigos éticos de comportamiento para las generaciones futuras (Polo, 2016). Es así como la ética ha estado presente en la formación de sociedades, naciones, Estados, gobiernos, ideologías, organizaciones e instituciones como parte de una evolución humana que busca constantemente una mejor actuación para sí misma y para todo su entorno. Restructuraciones en códigos éticos dieron lugar a que terminaran épocas violentas en la historia, como la Inquisición, que originó reformas en la Iglesia Católica, o el Absolutismo Monárquico, que fue reducido progresivamente 
mediante cuestionamientos éticos populares —a veces violentos—, los cuales condujeron a la formación de monarquías constitucionales y a la reconsideración de la democracia greco-romana (Mifsud, 1985). Nuevos patrones éticos también han procurado la búsqueda de ideologías más justas respecto a la distribución del poder y de la riqueza, lo cual ha dado nacimiento a conceptos idealistas que han hecho oposición a los fundamentos del realismo, con resultados significativos como la abolición de la esclavitud. La ética, como pilar de evolución humana y social, ha estado siempre presente en los avances tangibles e intangibles de la raza humana.

Por ende, la ética no ha estado ausente de las organizaciones militares, y desde el momento de su fundación en las primeras formas de Estado, las fuerzas militares de las naciones, imperios o países fueron configurando un proceder ético en la teoría y la práctica. Históricamente, los ejércitos más destacados estructuraron códigos éticos sólidos que incluso se señalaron como la columna vertebral de la disciplina y la moral de las tropas (Cifuentes \& Torres, 2019). El Imperio Romano, por ejemplo, demostró que gracias a sus códigos de comportamiento como sociedad logró mantener una supremacía fáctica durante más de mil años.

Asimismo, las dos guerras mundiales son referenciadas tradicionalmente como ejemplos de destrucción y ausencia de valores en las fuerzas militares que los enfrentaron. Mucho más visible en las naciones del eje por el holocausto judío causado por los alemanes y los crímenes de guerra realizados por Japón en la China ocupada, pero también han sido muy cuestionadas algunas acciones de los aliados, como el bombardeo de Hiroshima y Nagasaki, por parte de los Estados Unidos, y el de la ciudad de Dresde, por parte de los aliados en Europa (Serrano, 2018).

Luego de la Segunda Guerra Mundial, el impacto moral y ético que produjo el holocausto abrió el escenario internacional para la creación de la Organización de las Naciones Unidas. Con ello, la discusión ética adquirió mayor importancia dentro de las fuerzas militares, en cuanto al desarrollo de operaciones militares en concordancia con la estructuración del Derecho Internacional Humanitario como fundamento esencial de la humanización de la guerra (Rial, 2020). Desde entonces, las fuerzas militares no abandonan el 
interés ni la responsabilidad por implementar la ética en sus procedimientos militares en el mundo. Por el contrario, desde la Declaración Universal de los Derechos Humanos y la garantía progresiva de más derechos en el sistema jurídico internacional, las fuerzas militares de los países que no padecen dictaduras han procurado estudiar más a fondo el comportamiento ético de sus soldados en el ejercicio de sus acciones. Con el fin de que estas prácticas se articulen a la evolución del concepto de seguridad, con un enfoque sistémico que permite no perder de vista el potencial de las dimensiones humanas (Paez et al., 2018).

El Ejército Nacional de Colombia (2016a), en el Manual fundamental del Ejército MFE 1-01, aborda la ética como factor fundamental para establecer el buen trato entre líderes y soldados, y la establece como un elemento general concebido en la doctrina del Ejército que genera actuaciones dentro de los parámetros legales y legítimos en la ejecución operacional. En los fundamentos de formación del manual se infiere que la actuación idónea del personal determina que la doctrina se rige por la ética del Ejército y no al revés. Es decir, la ética se consolida como un principio fundamental con el cual incluso la construcción de la doctrina se debe enmarcar. El manual también relaciona la ética con la legitimidad y la credibilidad, pues indica que, para lograr un nivel de aceptación general ante el gobierno nacional y la población, se debe actuar de acuerdo con los mandatos de moral y transparencia.

Por otra parte, el Ejército Nacional (2016b), en el MFe 6-22 de liderazgo, también menciona la ética en la descripción del carácter como parte de los atributos que el líder debe poseer en el ejercicio de interacción con su entorno. Es así como el Ejército Nacional (2017a) en el Mre 1-0, establecido como piedra angular en el lanzamiento del proyecto Damasco, describe la ética militar como parte esencial de la profesión militar y señala que desempeñar con ética todas las actuaciones es uno de los principios fundamentales del Ejército. La ética también es mencionada en el numeral 11 del Código de Honor del Soldado, que dice: "La ética es mi única regla para tomar decisiones", y que aplica para miembros de la Fuerza de todo rango como guía personal de actuación personal y profesional. El término es mencionado específicamente en ese numeral, pero el código completo en sí con sus doce puntos es un referente ético comprensivo y holístico. 
Sin embargo, a pesar de que las fuerzas militares actuales han tenido procesos estructuradores de la ética en la profesión militar, estos esfuerzos son mayormente enfocados a la práctica, es decir, a la conducción de las operaciones militares (Toledo \& Vega, 2020). Es claro que el ejercicio de las acciones se consolida como el principal enfoque ético, pues es allí donde se encuentra la atención de la acción, buena o mala, a los patrones éticos que cada fuerza haya determinado para su organización, situación y personal específicos. Es decir, es en la práctica donde se hace observable la aplicación ética. Se es ético o no se es, luego de una observación ante hechos o acciones tomadas en un contexto, la ética sería solo un argumento teórico sin posibilidad de comprobación. De modo que los parámetros éticos en la doctrina militar no han sido mencionados directamente dentro de los procesos de planeamiento para el desarrollo de las operaciones militares, pues, al tener una concepción teórica, simplemente se relegan a la capacidad deductiva por parte del comandante militar en el terreno. Uno de los enfoques doctrinales en cuanto a planeamiento operacional es la rigurosidad metodológica de este proceso. No obstante, resulta efímero el análisis ético en cada parte del proceso militar para la toma de decisiones con respecto a la complejidad e incertidumbre que actualmente significa el ambiente operacional y estratégico (López et al., 2019).

\section{Método}

Se realiza una investigación documental, que de acuerdo con Galeano (2018) radica en obtener información no numérica, la revisión de archivos y el análisis de contenidos, que constituyen sus técnicas fundamentales; el término documentación se refiere a la amplia gama de registros escritos y simbólicos, así como a cualquier material y dato disponible.

La estrategia de investigación documental implica tratar de determinar los patrones subyacentes detrás de una serie de fenómenos, de modo que cada ocurrencia se vea como una referencia a un patrón potencial, refiriéndose al sentimiento, la visión, la percepción y la comprensión del evento o situación que se analiza. Por lo tanto, la descripción de los resultados encontrados y analizados se desarrolla en tres fases: (1) el proceso militar para la toma de 
decisiones, donde se exponen y analizan los conceptos y se realizan las descripciones del proceso; (2) el análisis de los modelos de ética aplicados al PMTD, y (3) el desarrollo e integración de modelos éticos al proceso militar para la toma de decisiones (РмтD).

\section{Resultados}

\section{El proceso militar para la toma de decisiones}

El proceso militar para la toma de decisiones es un recurso metodológico esencial con el que se busca entender una misión dentro de un contexto situacional propio y que tiene como producto final la construcción de documentos para guiar el desarrollo de las operaciones militares (U.S. Army, 2019a). El proceso es un prerrequisito esencial en la conducción de operaciones militares, ya que da el plan u orden de operaciones como productos finales y el respaldo doctrinal, operacional, jurídico y de mando con el cual la unidad militar puede realizar la misión (Ejército Nacional de Colombia, 2017b). La aplicación del proceso militar para la toma de decisiones se da en los niveles táctico y operacional en todas las fuerzas militares que, aunque sigan pasos y nombres distintos, se basan en los mismos principios metodológicos del arte del planeamiento. Se ha tomado el proceso militar para la toma de decisiones como el modelo más empleado y el que el Ejército Nacional de Colombia ha adoptado durante décadas en su doctrina militar. El modelo vigente en la doctrina militar colombiana consta de siete pasos (figura 1).

Los siete pasos de la figura 1 son reconocidos por la doctrina del Ejército Nacional en el manual Mre 5-0 (Ejército Nacional de Colombia, 2017b), que se establece como guía fundamental para los procesos de planeamiento tácticos y operacionales de la Fuerza.

Los procesos de planeamiento conjunto tienen una estructura similar en la que hay algunas variaciones hacia el desarrollo en un nivel estratégico-operacional con elementos más gerenciales y de coordinación respecto al proceso de planeamiento de las fuerzas JP 5-0 (Joint Chiefs Of Staff, 2020). Su definición, similar a la del proceso militar para la toma de decisiones, establece que 


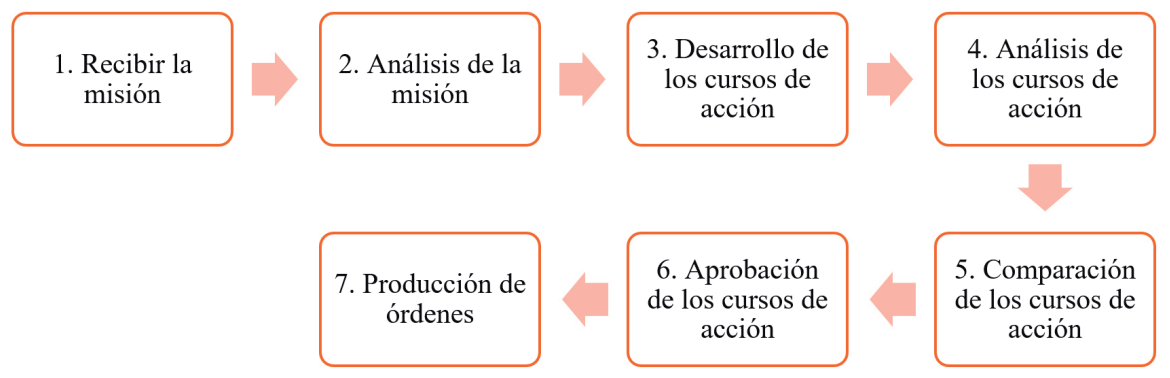

Figura 1. Pasos del proceso militar para la toma de decisiones. Fuente: elaboración propia con base en Wade (2015, pp. 1-2).

"es un proceso ordenado, analítico en una serie de pasos lógicos para enmarcar un problema, examinar una misión; desarrollar, analizar y comparar cursos de acción alternativos y seleccionar el mejor; y producir un plan u orden" (Wade, 2017). En la figura 2 se pueden apreciar los pasos para el proceso de planeamiento conjunto:

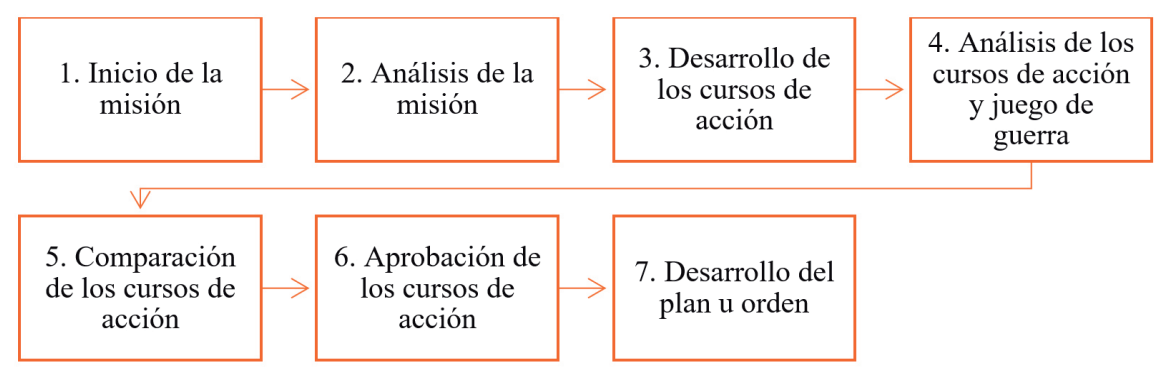

Figura 2. Pasos del proceso de planeamiento conjunto.

Fuente: elaboración propia con base en Wade (2017, pp. 3-69).

De acuerdo con el Ejército Nacional de Colombia (2017b), el proceso de planeamiento conjunto es realizado por estados mayores de unidades militares conjuntas que cumplen con el principio de comando único con disposición de medios militares de dos o más fuerzas para el desarrollo de la misión. Su desarrollo tiene un espectro más amplio debido a que se deben tener en cuenta consideraciones para más de una fuerza militar, y, por su nivel, las operaciones conjuntas se desarrollan regularmente en teatros más extensos territorialmente. 
Aunque puede haber excepciones, su complejidad operacional no se considera menor y mucho menos en el sentido en que las operaciones conjuntas pueden tener mayor implicación e interacción con el nivel estratégico.

Las operaciones militares ordenadas en planes de campaña, en planes y órdenes de operaciones revisten gran complejidad por el alcance que se da en los objetivos establecidos, cuya consecución demanda una coordinación y empeño de alta efectividad (Paparone, 2017). De acuerdo con el manual ATP 5-19 (U.S. Army, 2014), la complejidad de los procesos de planeamiento operacionales proviene del nivel de riesgo que enfrentan las propias tropas en su conducción. El riesgo se mide en factores de probabilidad e impacto, que tienen variaciones de acuerdo con la situación específica para cada misión. Se debe mencionar que la conducción de operaciones es el sorteo continuo de riesgos tratando de lograr los resultados más favorables hasta cumplir la misión. En suma, el riesgo es inherente a las operaciones militares, por lo que las fuerzas se preparan efectivamente para enfrentarlo.

Es así como el riesgo se hace una constante dentro del planeamiento de las operaciones militares en todos sus niveles (U.S. Army, 2014). Si bien un líder militar debe garantizar el cumplimiento de su misión manteniendo la integridad de sus hombres, ir a la guerra pretendiendo que no habrá afectación en el personal es irreal e incluso irresponsable. La efectiva gestión del riesgo permite al militar reducir las posibilidades de afectación hasta los niveles más bajos posibles. Por estas razones se establece que el apropiado manejo del riesgo permite una mejor toma de decisiones en el planeamiento de operaciones militares.

Mejores decisiones permiten desarrollar niveles de confianza y liderazgo, lo que en consecuencia genera mayor soporte y efectividad ante el éxito en las misiones. En otras palabras, planear teniendo el manejo del riesgo incluido en el proceso da una sensación de seguridad que hace tomar decisiones más firmes y seguras. El general Dennis J. Reimer, del Ejército estadounidense, declaró en 1995 que el manejo del riesgo es un multiplicador de combate al preservar el poderío de la fuerza, brindar flexibilidad y ser un elemento de total integración en el proceso militar para la toma de decisiones (U.S. Army, 1998).

Por eso, el campo de aplicación del manejo del riesgo llega a todos los procesos del ejército y es útil en el empleo de su fuerza total, así como de 
las fuerzas conjuntas (U.S. Army, 1998). Su implementación en los procesos militares ha ofrecido grandes beneficios, pues ha permitido mitigar los peligros en las operaciones militares altamente complejas ejecutadas por el Ejército de los Estados Unidos (U.S. Army, 2001). Con esta premisa, los norteamericanos han logrado conservar vidas y recursos, identificar medidas de control y proporcionar alternativas razonables para llegar al cumplimiento de una misión.

El mundo empresarial se vale de estas lecciones y reconoce que tener un adecuado control sobre los riesgos le permite tomar acciones más productivas y rentables (Yang \& Gabrielsson, 2017). Las empresas con mayor capital toman riesgos con una mayor confianza en comparación con las que tienen un capital limitado. Esto, traducido en términos militares, es equivalente a que una unidad militar más entrenada puede arriesgarse más que una que posee un adiestramiento básico. Por ejemplo, una unidad de fuerzas especiales decidirá y ejecutará más fácilmente cruzar un río que una unidad de tropas de soldados en servicio militar. En términos de dirección ocurre lo mismo, pues los gerentes empresariales no toman decisiones de peso en sus negocios basándose solamente en la información que está disponible públicamente (Koerner \& Staller, 2021). Para ello, se esfuerzan por obtener información relevante, crítica y actualizada que los lleve a tomar las mejores decisiones para su negocio. Llevado al esquema militar, las operaciones militares deben tener el respaldo de una información de inteligencia robusta que permita tomar los riesgos necesarios en su ejecución y así cumplir el objetivo.

Debe tenerse en cuenta que el análisis de los riesgos no puede tomarse de forma general: cada unidad militar puede analizar de forma diferente los riesgos según sus condiciones y características. Es así como el manejo del riesgo en la toma de decisiones se hace progresivo (Martínez, 2010). Es decir, a medida que se asumen riesgos se adquiere la capacidad para asumir otros mayores. El manejo del riesgo siempre ha influido el comportamiento humano dividido entre emocionalidad y racionalidad, una como las sensaciones y otra como el análisis pragmático. Elementos humanos como la intuición, el instinto y el presentimiento han regulado las decisiones humanas para llevar a la raza a su preservación y supervivencia. Pero su evolución ha llevado el manejo del riesgo al campo militar, cuyo aporte ha generado la toma de decisiones en un sentido 
analítico y procesal o metódico, basándose en experiencias que han producido ciertos procedimientos.

Sin embargo, la parte sensorial no podrá ser nunca descartada del proceso de toma de decisiones, pues en determinados momentos el líder debe recurrir a ella cuando sea necesario que tome decisiones inmediatas (Marr, 2001). De hecho, el líder militar de las unidades en el terreno se enfrentará a la toma de decisiones de momento inmediato, cuando tendrá que recurrir a su instinto porque no tiene tiempo para procedimientos. Es por esta razón que los planeadores militares deben estudiar el riesgo dentro del proceso de planeamiento con metódico análisis y debido margen temporal. De esta manera se consigue un criterio mecánico eficiente para la toma de decisiones en el tiempo y la circunstancia que se requiera. En síntesis, el adecuado manejo del riesgo permite tener mayores niveles de confianza y, por consiguiente, que se puedan tomar decisiones de mejor calidad dentro del proceso militar. Saber a qué se enfrentan las tropas y qué consecuencias se pueden llegar a presentar afianza la efectividad decisional del planeador militar y por ende la efectividad en la conducción de las operaciones.

Lo anterior aún no menciona la inclusión de la ética en el proceso militar para la toma de decisiones, precisamente porque los manuales actuales no la incluyen específicamente al procedimiento. En la figura 3 se plantearon y resaltaron los elementos actuales que doctrinalmente interactúan en los procesos de planeamiento como esenciales para su desarrollo exitoso, con el fin de analizarlos y direccionarlos hacia la ética. En esta se incluyen elementos conjuntos y de fuerza con el propósito de unificar el análisis con los procesos de planeamiento en general. El arte operacional y el mando tipo misión son los elementos cognitivos e intangibles del planeador militar que tienen que complementar el proceso con base en elementos no definibles ni científicamente observables como el talento, la experiencia, el análisis, el criterio, la coherencia, la malicia, entre otros (U.S. Army, 2019a). 


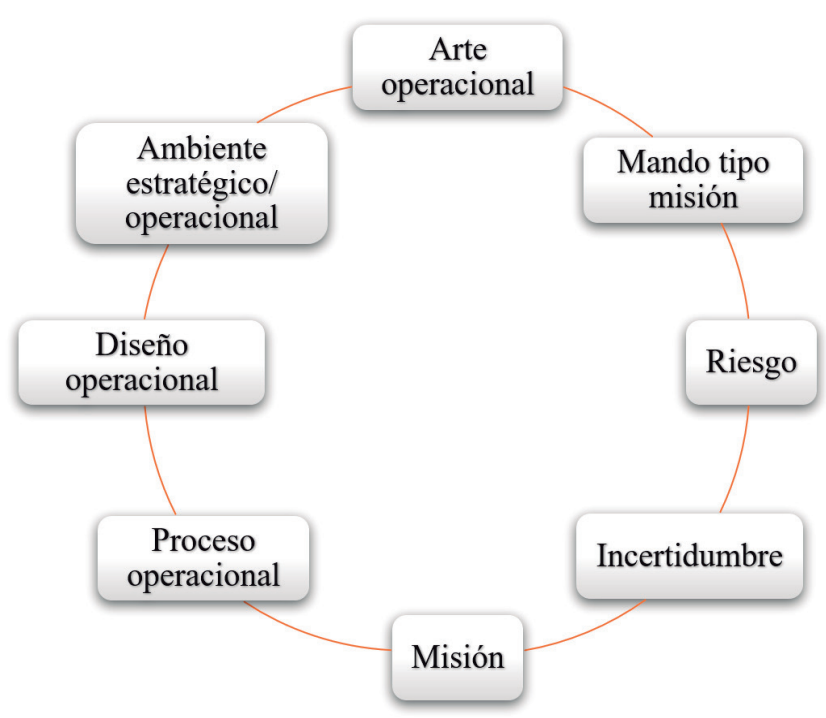

Figura 3. Elementos de interacción en los procesos de planeamiento.

Fuente: elaboración propia con base en U.S. Army (2012; 2019a), Ejército Nacional de Colombia (2017b) y Joint Chiefs Of Staff (2020).

El ambiente estratégico y operacional es el entorno en el que se desarrollarán las operaciones. En este encontraremos la complejidad mencionada en el mundo actual. El diseño y proceso operacional conforman la doctrina o la ciencia medible que todo planeador militar debe tener como parte de la técnica en la realización del proceso. La misión es única e irrepetible, por lo cual una misión nunca podrá ser usada dos veces. Aun cuando se ejecute una misión aparentemente similar, el solo hecho de que no sea en el mismo tiempo la hace diferente, por lo tanto cada misión es única y se ejecuta una sola vez.

Teniendo en cuenta los elementos de la figura 3, los manuales de planeamiento militar generalmente se enfocan en el cumplimiento de la metodología doctrinal del proceso en sí, como base sustancial de la producción de documentos finales de calidad, cuya terminación de acuerdo con parámetros técnicos se define como el índice de calificación cualitativa al término del proceso. Es decir, los productos del proceso de planeamiento serán catalogados como "buenos" o de "alta calidad" si se hicieron con esquemas de paso a paso y se cumplió de alguna manera con cada uno de ellos. Esto deja 
por fuera el contexto ético que debe tener cada uno de los pasos en el proceso militar para la toma de decisiones, por lo que se argumenta la existencia de un vacío ético-doctrinal en el enfoque de planeamiento de las operaciones. Ya fue mencionado que las organizaciones militares poseen doctrina que aborda la discusión ética de la profesión militar en el contexto general, pero se identifica directamente en manuales de liderazgo o de ética (Ejército Nacional de Colombia, 2017a).

Al momento de abordar los manuales de procesos de planeamiento, la doctrina no es clara en definir cómo se aplica el código ético dentro del proceso. El Adrp 5-0 U.S. Army (2012) contiene su proceso de planeamiento, pero no hace menciones específicas de la ética en los procedimientos. El Joint Chiefs Of Staff (2020) de las Fuerzas Militares estadounidenses no hace menciones éticas en su contenido. En el caso colombiano pasa lo mismo. El manual MFE 5-0 (Ejército Nacional de Colombia, 2017b) no contempla la ética en el detalle del proceso. De hecho, el proceso de planeamiento no introduce parámetros analíticos sobre la ética, lo que sin duda ha aportado a que se presenten errores evitables en la conducción y el desarrollo de las operaciones.

Según lo anterior, el riesgo está asociado al nivel de incertidumbre con el que se lidia en las operaciones. Esto indica que la ética tiene un papel fundamental con respecto a estos dos elementos a tener en cuenta en el proceso de planeamiento, toda vez que en situaciones de gran incertidumbre y riesgo se generan dilemas éticos que por su ausencia en la metodología simplemente no son, por lo menos, pensados (Raglin, 2019).

\section{Modelos de ética para el proceso de planeamiento militar}

Es realmente conveniente establecer modelos para identificar cómo podría mejorarse el sentido ético en los procesos de planeamiento. Para ello, se debe comenzar definiendo que un dilema ético es "una situación en la cual el decisor tiene un conflicto moral en el que entran en choque valores y virtudes entre sí, y dos o más soluciones tienen similar apoyo y mérito moral" (Kem, 2006, p. 25). Chang (2014, mayo) describió que lo que hacía una decisión difícil de tomar era el peso equilibrado entre las dos opciones, de modo que se hacía complejo identificar cuál de las dos era mejor o peor que la otra, el 
punto determinante era no tener una opción visiblemente más favorable que la otra. De igual forma, Kem (2006) resalta que, si un decisor ético es puesto frente a dos opciones y una de ellas es claramente ética mientras que la otra no, entonces no hay dilema. Por ello los dilemas éticos se presentan cuando existe un grado de relativismo en las dos o más opciones que se presentan haciendo que todas tengan una imagen de "correctas" y ninguna de incorrecta.

\section{El triángulo ético en la toma de decisiones militar}

El manual de liderazgo del Ejército de Estados Unidos (Center for Army Leadership, 2004) contiene un modelo ético de toma de decisiones con cuatro puntos: (1) Definir el problema, (2) Conocer las reglas relevantes, (3) Desarrollar y evaluar los cursos de acción y (4) Escoger el curso de acción que más representa los valores del ejército.

El modelo es tomado como simplista debido a que carece de explicación en su descripción y forma de aplicación, de manera que deja el contexto subjetivo demasiado abierto a interpretaciones. De por sí los asuntos éticos tienen un alto contenido de subjetividad, sin embargo, la ausencia de explicaciones y descripciones puede hacerlo mucho más profundo. El modelo de Kem (2006) identifica problemas sustanciales en cada paso. En la definición del problema el manual no aborda cómo se define un problema. En los asuntos de análisis estratégicos y el método científico, la definición del problema es el paso de mayor complejidad. En este caso, el manual no aborda su relación con los factores causales, que son un elemento clave para definir el problema en cualquier contexto.

Por otra parte, "conocer las reglas relevantes" es una oración con mucha amplitud, ya que estas pueden ser interpretadas de diferentes maneras según el ambiente, región o nación donde se esté. Una fuerza multinacional tendría un mayor espectro de reglas si se encuentra en un territorio extranjero y no todas las reglas locales podrían ser aplicables a la misión. El desarrollo, evaluación de los cursos de acción y la posterior selección del más apropiado son procesos más familiares a los planeadores militares, pero en el encuentro con dilemas éticos no existe un método de toma de decisiones que permita descartar las opciones inapropiadas cuando existe una fricción de valor moral sobre ellas. 
Finalmente, el manual Adp 6-22 (U.S. Army, 2019b) ya no incluye el modelo de toma de decisiones éticas. En su lugar, contiene un párrafo en el que describe el razonamiento ético y otro en el que habla de las órdenes éticas. En la figura 4 se diagrama el modelo de triángulo ético para la toma de decisiones basado en Kem (2006).

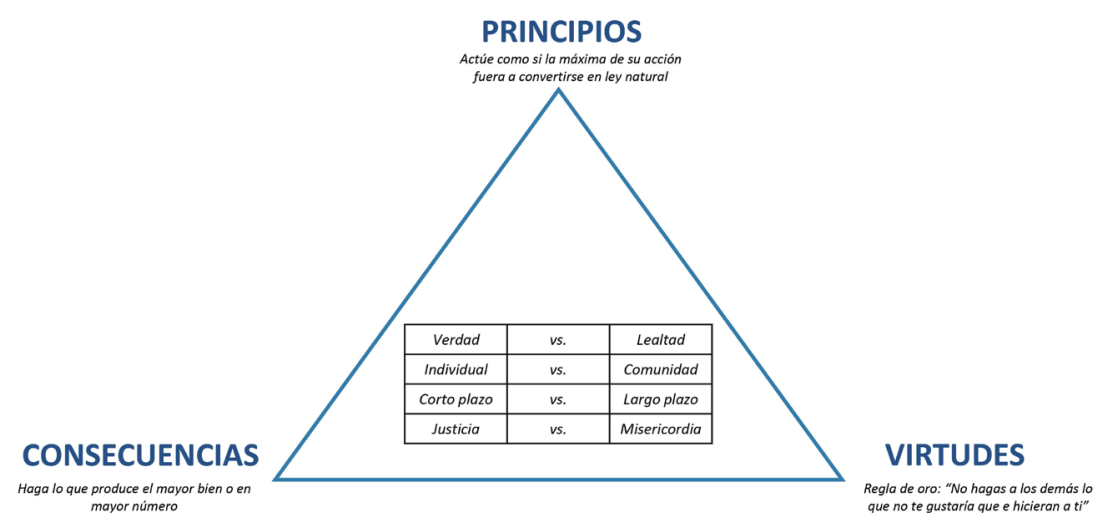

Figura 4. Triángulo ético para la toma de decisiones militares.

Fuente: elaboración propia con base en Kem (2006, p. 33).

El triángulo indica la toma de decisiones basándose en tres referencias de toma de decisión fundamentada en: principios, virtudes y consecuencias. De acuerdo con Kem (2006), es recomendable el empleo del modelo de proceso ético militar para la toma de decisiones, excluyendo el segundo paso, pero en su lugar, aplicando el modelo del triángulo ético en el cual se hace un balance entre principios, virtudes y consecuencias. Los dilemas éticos se resumen en cuatro diferentes tipos: verdad vs. lealtad, individual vs. comunidad, corto plazo $v s$. largo plazo y justicia $v s$. misericordia. El análisis de los cursos de acción a través del lente de este modelo, tomando primero los principios, luego las consecuencias y finalmente las virtudes, ayudaría a observar mejor la diferencia entre opciones que representen dilemas éticos.

En la mayoría de las ocasiones, los dilemas éticos presentan fricciones entre los principios y las virtudes. Sin embargo, cuando se contrastan con las potenciales consecuencias, estas tienden a determinar las decisiones (Kem, 2006). Cuando las consecuencias son igualmente impactantes, los principios 
o los valores marcarían la diferencia. Este modelo representa un sistema de pesos y contrapesos o check and balances, tal cual como funciona en la democracia. Es comparable a una división de poderes en el razonamiento en donde todos los elementos no permiten que uno solo de ellos gobierne las decisiones. De una manera objetiva se balancean y complementan, con lo cual dan lugar a un equilibrio de razonamiento ético y se obtiene un marco adaptable a cada situación sin necesidad de códigos que difícilmente se acoplan a diversas situaciones.

\section{Medición de la incertidumbre como herramienta de anticipación a dilemas éticos}

La incertidumbre está relacionada con la decisión ética, en la medida en que cuanto mayor sea el nivel de incertidumbre, menores serán las probabilidades de tomar decisiones éticas inmediatas en el terreno durante las operaciones militares (Lipshitz \& Strauss, 1997). Esto se debe a que la incertidumbre se da en cantidades bajas de información respecto a un sujeto, un lugar, un tiempo o una condición que las unidades militares enfrentarán en el desarrollo de la misión. Con mayor cantidad de información se tienen mejores probabilidades de tomar decisiones éticas adecuadas, pues no existen vacíos que creen confusión o dilemas éticos.

Según lo anterior, la incertidumbre en el proceso militar para la toma de decisiones es identificada en el análisis del riesgo (Vertzberger, 1998). Este se mide mediante un método que les muestra a los planeadores militares, de forma cualitativa o cuantificada, el nivel de riesgo que se tiene en determinada situación de la operación que se planea. El manejo del riesgo en el proceso militar para la toma de decisiones inicia en el subpaso 7, "Iniciar manejo del riesgo", del paso 2, “Análisis de la misión” (Von Benken, 2017). Por otro lado, U.S. Army (2014) describe el proceso de manejo del riesgo en cinco pasos cíclicos, que se describen en la figura 5.

El proceso pretende mitigar el riesgo, de manera que se obtiene un riesgo residual o riesgo que no se puede minimizar y para el cual la única opción es implementar acciones para evitar su ocurrencia. Su aplicación se recomienda de forma continua y paralela durante el resto del proceso de planeamiento e 


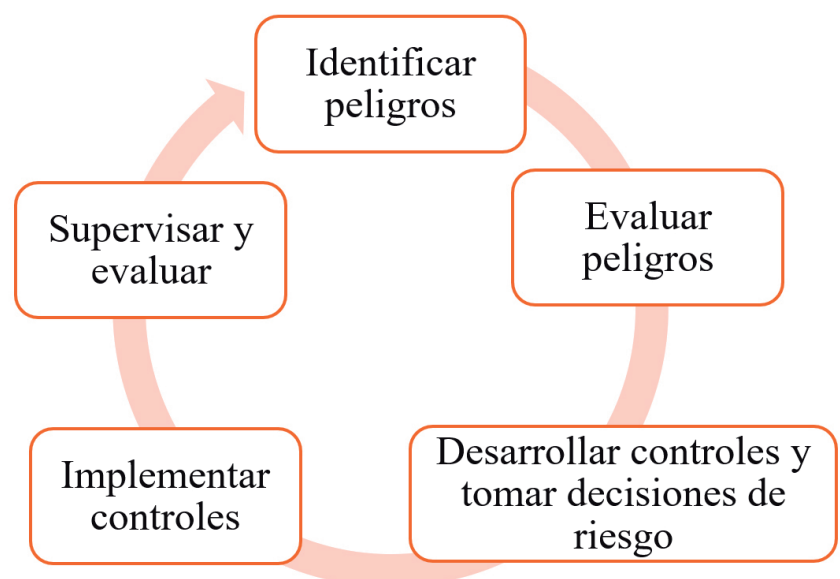

Figura 5. Pasos del proceso de manejo del riesgo.

Fuente: elaboración propia con base en U.S. Army (2014, p. 3-59).

incluso después de que las unidades se encuentren cumpliendo la misión. La medición del riesgo es un proceso permanente.

Se han mencionado diferentes elementos en los procesos operacionales y tácticos. Por otra parte, en el contexto estratégico también ha habido teóricos que han propuesto métodos para manejar la incertidumbre y el riesgo en la toma de decisiones. Dean y Sharfman (1993) desarrollaron cinco hipótesis para medir la incertidumbre en la toma de decisiones militares en el nivel estratégico:

1. La amenaza competitiva será positivamente relacionada con el procedimiento racional en la toma de decisiones estratégicas.

2. La importancia de decisiones estratégicas será positivamente relacionada con el procedimiento racional.

3. El control externo será positivamente relacionado con el procedimiento racional en la toma de decisiones estratégicas.

4. La contención de objetivos será negativamente relacionada con el procedimiento racional en la toma de decisiones estratégicas.

5. La incertidumbre será negativamente asociada con el procedimiento racional en decisiones estratégicas. 
Las cinco hipótesis muestran que hay una relación entre la necesidad de supervivencia (hipótesis 1), la importancia de la misión (hipótesis 2), el mando y control (hipótesis 3), la defensa (hipótesis 4) y, finalmente, el nivel de riesgo en la quinta hipótesis, la cual se vincula de forma negativa por las probabilidades contraproducentes que tiene. Por lo tanto, también en el nivel estratégico, la toma de mejores decisiones está relacionada con lograr minimizar el nivel de incertidumbre que se tenga en una situación determinada (Dean $\&$ Sharfman, 1993). A mayor nivel de incertidumbre, menor probabilidad de acierto, y a menor nivel de incertidumbre, mayor probabilidad de acierto en la toma de decisiones.

\section{Integración de modelos éticos al PMTD}

El modelo del triángulo ético y la medición de incertidumbre para anticipar dilemas éticos presenta un marco ético inicial y apropiado para complementar el proceso militar para la toma de decisiones (Kem, 2006). El primero podría ser empleado en los pasos de desarrollo, comparación y análisis de los cursos de acción, así como en el juego de guerra, aunque el desarrollo de los cursos de acción, dependiendo del nivel de la guerra en que se esté planeando, son de carácter general respecto a la misión. Es decir, las acciones o situaciones subsecuentes que se presentan serán concebidas solo en una ramificación inicial y no solo serían desarrolladas en profundidad cuando el tiempo lo permite, lo cual, en épocas de crisis, no es muy común. Pero la aplicación aun en términos generales a los cursos de acción daría un criterio ético sustancial que ayudaría a tomar el curso de acción más adecuado a los valores de la fuerza militar individual o conjunta que desarrolle el proceso.

La medición de la incertidumbre permitiría prever qué situaciones podrían presentarse en el desarrollo de los cursos de acción de una manera más particular, con el fin de anticipar situaciones que puedan suponer un dilema ético para las unidades en el terreno (McDermott et al., 2003). El logro de una efectiva anticipación obligaría a los estados y planas mayores a buscar más información con el fin de disminuir el nivel de incertidumbre en determinada situación y así reducir las posibilidades de dilemas éticos en el terreno. En el peor de los casos, de no lograrse información suficiente, al menos las 
tropas podrían ser informadas de la incertidumbre en determinado punto de su misión y esto haría que tengan mayor precaución ante los posibles eventos.

Los planeadores militares deben analizar durante el desarrollo del proceso militar para la toma de decisiones si están causando dilemas éticos a las unidades militares y/o sus comandantes por fallas en su proceso de planeamiento. La provisión de información clave relacionada con las decisiones éticas en los documentos productos del proceso de planeamiento, como la anteorden o la orden de operaciones, es esencial para disminuir el riesgo de dilemas éticos por parte de las unidades militares en el terreno.

Sin embargo, la determinación de la manera de aplicar la ética en el proceso militar para la toma de decisiones podría definirse de otra manera en que el proceso es demasiado amplio y complejo como para hacer inferencias éticas más allá del desarrollo adecuado del proceso mismo, ya que podría argumentarse que sería muy difícil anticipar los dilemas éticos que las unidades militares encontrarán en el terreno porque existen millones de posibilidades. Es decir, que un planeador militar estaría actuando éticamente de manera suficiente mientras siga los pasos del proceso como lo dicta la doctrina. Algunos altos mandos militares han manifestado que la ética en la doctrina del proceso de planeamiento conjunto se encuentra incluida en la forma como se diseñó su procedimiento y que el proceso, de seguirse doctrinalmente, es un acto ético en sí, y que por lo tanto es debido a ello que no hay menciones éticas específicas en los manuales de planeamiento.

El proceso militar para la toma de decisiones es igualmente tan amplio y complejo, que podrían desarrollarse preguntas de análisis para cada uno de los pasos, por ejemplo, ¿cuál es el referente ético para al análisis de la información del adversario en un proceso de planeamiento? o ¡cómo implementar la aplicación de la ética en las matrices de toma de decisiones?

La complejidad del proceso de planeamiento reviste ambigüedades, tal como que en determinadas situaciones sería apropiadamente ético realizar todos sus pasos en forma minuciosa, mientras que en otras situaciones más urgentes y de acción rápida, lo más ético podría ser una ejecución resumida, obviando pasos innecesarios para la misión. Este es el nivel de complejidad y responsabilidad que enfrentan los planeadores militares y los líderes de las 
unidades en el terreno. Es por ello que es necesario continuar realizando análisis y discusiones del tema ético en los procesos de planeamiento con el fin de construir una fuerza más robusta y legítima.

La falta de concepciones éticas específicas para el proceso militar para la toma de decisiones es un vacío importante que puede causar una transferencia del riesgo y del dilema ético desde las planas y estados mayores hacia las unidades militares en el terreno. Esto puede acentuarse debido a las condiciones críticas en las que se desempeñan y que generan una menor capacidad de análisis objetivo en el momento de actuar en circunstancias de crisis y estrés, en los cuales las decisiones deben ser tomadas muchas veces de forma inmediata bajo presión de la situación (Beaulieu-B \& Dufort, 2017). Tendrían que enfrentar los soldados (de todo rango) en el terreno dilemas éticos tácticos, muy probablemente en condiciones de hostilidad, alto riesgo, estrés y fatiga, razón por la cual tendrían mayores probabilidades de actuar equívocamente. Por una falta de análisis ético en los procesos de planeamiento, las unidades militares cometerían acciones en contra del marco de actuación adecuado, lo cual causaría que las operaciones militares pudieran ser cuestionadas legal, legítima y jurídicamente. Por tanto, se produce un entorno desfavorable para la profesión militar respecto a su compromiso ético.

Aunque las planas y estados mayores también tienen un trabajo arduo y crítico en el desarrollo de las operaciones al realizar su planeamiento, sus condiciones de análisis ético son mucho más favorables que los de las unidades militares que ejecutan dichas operaciones (De Graaff et al., 2017). Generalmente se encuentran en instalaciones militares con menos intensidad de riesgo que las unidades que están en la primera línea de acción o de batalla y quienes verdaderamente sienten la presión, el estrés y el riesgo a partir de la línea de partida.

\section{Conclusión}

Así como crece la capacidad de las Fuerzas, crece el nivel de exigencia y control por parte de organismos nacionales e internacionales y de la población en general para asegurar un desempeño profesional, pero ante todo ético. No 
obstante, no solo se trata de control civil, también se cuenta con un tendiente esfuerzo hacia la deslegitimación de las fuerzas militares y sus nobles labores por parte del activismo antimilitarista y comulgantes de organizaciones o grupos generadores de riesgo e incertidumbre.

Los actuales teatros estratégicos y operacionales presentan las mismas características en cuanto a complejidad e incertidumbre. Mayor número de actores y desafíos hacen que las fuerzas militares estén cada vez mejor preparadas y esto incluye la exigencia de un marco de actuación ético robusto. Luego de la Segunda Guerra Mundial y la posterior definición de la humanización de la guerra a través de la Declaración Universal de los Derechos Humanos, la ética se ha venido inculcando con marcado progreso en la doctrina. Sin embargo, resulta ser una tarea inacabada, ya que ha tenido un enfoque tradicionalmente presuntuoso en la estructuración profesional de las fuerzas armadas, con gran énfasis en las áreas del liderazgo.

Su ausencia se observa de manera clara en el proceso militar para la toma de decisiones. La doctrina existente para el planeamiento militar no contempla de manera específica a la ética y no se determina cómo el planeador militar puede ser ético o no. Precisamente, esto ha generado un vacío ético en sí mismo al asumir generalmente que el seguimiento a la metodología establecida para el planeamiento brinda el marco ético suficiente. La situación empeora si se tiene en cuenta que hoy los procesos y procedimientos se encuentran forzados a afrontar situaciones y misiones cada vez menos tradicionales.

Los modelos existentes, como el triángulo ético de Kem (2006) o el relacionamiento del análisis del nivel de incertidumbre al nivel de decisiones éticas en la toma de decisiones, aportan un marco de aplicación adaptable al modelo actual. La consideración de las consecuencias, los principios y los valores en el triángulo ético de Kem (2006), al momento de tomar una decisión, es un ejemplo de que en los pasos que se siguen en el proceso de planeamiento pueden incluirse análisis de rigor que el planeador militar debe ejecutar con el fin de asegurarse en lo máximo posible que una decisión tomada no cause una transferencia del dilema ético hacia las unidades en el terreno. Asimismo, la concientización en el planeador militar de que un plan operacional con mayor nivel de incertidumbre — que pudo ser disminuida en el proceso de planea- 
miento- entregado a las unidades de maniobra causará mayor probabilidad de riesgo ético en las decisiones inmediatas que les exija la misión.

Se enfatiza en que la necesidad de inclusión de un marco ético más específico en el proceso de planeamiento militar no debe tomarse como una exigencia de cambio, ni una previsión imposible de lograr en un proceso altamente subjetivo y dependiente de muchos actores y variables. Se trata de mejorar los niveles de análisis por parte de los planeadores militares con el fin de que tengan una proyección ética prospectiva que permita evitar cuestionamientos éticos a la Fuerza. Un ejemplo de ello es la inclusión de ejercicios de simulación sobre ética en el juego de guerra, además de los elementos de acción-reacción y contra-reacción operacionales que sin duda podrían prevenir eficientemente situaciones de dilemas éticos en el terreno en las que las unidades por presiones de tiempo y lugar podrían actuar equivocadamente.

Finalmente, es necesario entender que un proceso militar para la toma de decisiones con mayores alcances éticos en el desarrollo de sus pasos brindaría mayor legitimidad, mayor respaldo y mayor efectividad operacional de las tropas militares, traducible en beneficios estratégicos de la defensa de la democracia y el mismo Estado.

\section{Referencias}

Alvinius, A., Ohlsson, A., \& Larsson, G. (2018). Organizational challenges and leaders' coping strategies: A qualitative study of Swedish military staff organization. Journal of Military Studies, 9(1), 24-33. https://doi.org/10.1515/jms-2017-0002

Aristizábal, M., J. C., Páez, M., C. A., \& Quintero L., A. M. (2020). Procesos de calidad en la educación militar. Reflexiones en torno a la formación en investigación en la Escuela de Armas Combinadas. En L. A. Albornoz, A. E. Fernández \& C. A. Betancur (eds.), Práctica pedagógica en el Ejército Nacional de Colombia: Reflexiones en torno a la formación, gestión educativa y la investigación (pp. 159-180). Sello Editorial ESMIC. https://doi.org/10.21830/9789585241459

Beaulieu-B, P., \& Dufort, P. (2017). Introduction: Revolution in military epistemology. Journal of Military and Strategic Studies, 17(4). 2-20. https://jmss.org/article/view/58252/43818

Center for Army Leadership (2004). The U.S. Army leadership field manual: Battle-tested wisdom for leaders in any organization. McGraw-Hill.

Chang R. (2014, mayo). How to make hard choices [Conference, TED Ideas worth spreading TEDSalonNY2014]. https:/www.ted.com/talks/ruth_chang_how_to_make_hard_choices 
Cifuentes Medina, J. E., \& Torres Ortiz, J. A. (2019). Reflexiones en y para la enseñanza de la historia de la ética. Hallazgos, 16(31), 167-186. https://doi.org/10.15332/s17943841.2019.0031.07

Cook, M. L. (2000). Moral foundations of military service. The US Army War College Quarterly: Parameters, 30(1), 117-29. https://press.armywarcollege.edu/parameters/vol30/iss1/3/

De Graaff, M. C., de Vries, P. W., Van Bijlevelt, W. J., \& Giebels, E. (2017). Ethical leadership in the military: The gap between theory and practice in ethics education. En P. H. J. Olsthoorn (ed.), Military ethics and leadership (pp. 56-85) [International Studies on Military Ethics, 3]. Brill. https://doi.org/10.1163/9789004339590_004

Dean, J. W., \& Sharfman, M. P. (1993). Procedural rationality in the strategic decision区 making process. Journal of Management Studies, 30(4), 587-610. https://doi. org/10.1111/j.1467-6486.1993.tb00317.x

Ejército Nacional de Colombia. (2016a). Manual Fundamental del Ejército MFE 1-01. Doctrina. Imprenta Militar del Ejército.

Ejército Nacional de Colombia. (2016b). Manual Fundamental del Ejército MFE 6-22. Liderazgo. Centro de Doctrina del Ejército (CEDOE).

Ejército Nacional de Colombia. (2017a). Manual Fundamental del Ejército MFE 1-O. El Ejército. Centro de Doctrina del Ejército (CEDOE).

Ejército Nacional de Colombia. (2017b). Manual Fundamental del Ejército MFE 0-5. Proceso de Operaciones. Imprenta Militar del Ejército.

Fotion, N., \& Elfstrom, G. (1986). Military ethics: Guidelines for peace and war. Routledge Kegan \& Paul.

Galeano M., M. E. (2018). Estrategias de investigación social cualitativa. Fondo Editorial FCSH.

González, J. (1999). Ética. Revista CONAMED, 4(3), 11-16. https://www.medigraphic.com/ $\mathrm{pdfs} /$ conamed/con-1999/con993e.pdf

Joint Chiefs of Staff. (2020). JP 5-0 Joint Planning. https://fas.org/irp/doddir/dod/jp5_0.pdf

Jordán, J. (2016). La técnica de construcción y análisis de escenarios en los estudios de seguridad y defensa. Análisis GESI, 24(1), 28.

Kem, J. D. (2006). The use of the "ethical triangle" in military ethical decision making. Public Administration and Management, 11(1), 22-43.

Koerner, S., \& Staller, M. S. (2021). From data to knowledge. Training of police and military special forces in systemic perspective. Special Operations Journal, (7), 29-42. https://doi. org/10.1080/23296151.2021.1904571

Lipshitz, R., \& Strauss, O. (1997). Coping with uncertainty: A naturalistic decision-making analysis. Organizational Behavior and Human Decision Processes, 69(2), 149-163. https://doi.org/10.1006/obhd.1997.2679

López, C., Puga, C., Yépez, J., \& Maiguashca, J. (2019). La toma de decisiones en el campo militar, basado en información geo-espacial temática para la seguridad y defensa en Ecuador. Revista de Ciencias de Seguridad y Defensa, 4(8), 47-54. 
Marr, J. J. (2001). The military decision making process: Making better decisions versus making decisions better [Monografía, School of Advanced Military Studies United States Army Command and General Staff Colleg]. Defense Technical Information Center. https:// apps.dtic.mil/sti/citations/ADA387136

Martínez Velasco, G. P. (2010). Incorporación de la gestión de riesgos en la formación militar. [Monografía de diplomado, Instituto de Altos Estudios Nacionales]. Repositorio IAEN. http://repositorio.iaen.edu.ec/handle/24000/435

McDermott, P., Hutchins, S., Barnes, M., Koenecke, C., Gillan, D., \& Rothrock, L. (2003, octubre). The presentation of risk and uncertainty in the context of national missile defense simulations. Proceedings of the Human Factors and Ergonomics Society Annual Meeting, 47(3), 562-566. https://doi.org/10.1177/154193120304700365

Mifsud, S. J. A. (1985). El texto "Evangelio, ética y política” y su status epistemológico. Revista de Ciencia Politica, 7(1), 115-119.

Muñoz, F., A. M. (2013). El uso de la fuerza en el marco de los DDHH, un nuevo reto para las Fuerzas Militares en Colombia [Tesis de Especialización, Universidad Militar Nueva Granada]. Repositorio de la Universidad Militar Nueva Granada. https://repository.unimilitar.edu.co/handle/10654/11227

Paez, C. A., Peón, I. E., \& Ramírez, Y. (2018). Contexto de la seguridad ciudadana en América Latina y el Caribe: revisión de literatura (2007-2017). Revista Científica General José María Córdova, 16(24), 83-106. https://dx.doi.org/10.21830/19006586.360

Paparone, C. (2017). How we fight: A critical exploration of US military doctrine. Organization, 24(4), 516-533.

Park, S. (2018). Globalization and the incremental impact on the security and defense sector. En R. C. Kloosterman, V. Mamadouh \& P. Terhorst (eds.), Handbook on the Geographies of Globalization (pp. 318-328). Edward Elgar Publishing.

Polo Santillán, M. Á. (2016). Ética: definiciones y teorias. Fondo Editorial Universidad de Lima.

Raglin, A. (2019, julio). Presentation of information uncertainty from IoBT for military decision making. En N. Streitz \& S. Konomi (eds.), Distributed, ambient and pervasive interactions. Conference Proceedings HCII 2019 (pp. 39-47). Springer, Cham. https://doi. org/10.1007/978-3-030-21935-2

Rayón, M. C. (2018). La globalización: su impacto en el Estado-Nación y en el derecho. Revista Jurídica Derecho, 7(8), 19-37.

Rial, J. A. (2020). Sobre la creación de las Naciones Unidas. Boletín del Departamento de Seguridad y Defensa del IRI, 36, 10-11.

Serrano, A. J. M. (2018). El paradigma de la guerra en el siglo XX: ¿Instrumento de cambio? Revista Cientifica General José María Córdova, 16(23), 23-42. http://dx.doi. org/10.21830/19006586.305

Toledo A. M. P., \& Vega P., D. J., (2020). Consideraciones estratégicas para la implementación del arte y diseño operacional en la planificación y conducción de las operaciones militares en el Ejército ecuatoriano. [Tesis de maestría, Universidad de las Fuerzas Armadas]. Repositorio ESPE. http://repositorio.espe.edu.ec/handle/21000/22613 
Travis, D. S. (2019). Pursuing civilian control over the military. Armed Forces \& Society, 45(3), 546-560. https://doi.org/10.1177/0095327X18781539

U.S. Army. (1998). FM 100-14 Risk Management. Headquarters Department of the Army.

U.S. Army. (2001). FM 3-100.12 Risk Management. WVNavy LLC.

U.S. Army. (2012). Army doctrine reference publication (ADRP) 5-O. The Operations process. Createspace Independent Publishing Platform.

U.S. Army. (2014). Risk Management ATP 5-19. Headquarters Department of the Army.

U.S. Army. (2019a, julio 31). Army Doctrine Publication ADP 5-0 The Operations process. Headquarters Department of the Army.

U.S. Army. (2019b, noviembre 25). Army Doctrine Publication ADP 6-22 Army leadership and the profession. Headquarters Department of the Army.

Valdés G., P. (2020). La dirección de la guerra: Conducción operacional y gobierno politico de las operaciones militares [Tesis de doctorado, Universidad de Navarra].

Vertzberger, Y. Y. (1998). Risk taking and decision making: Foreign military intervention decisions. Stanford University Press.

Von Benken, D. J. (2017). Averse to initiative: Risk management's effect on mission command [Monografía, School of Advanced Military Studies United States Army Command and General Staff Colleg]. Defense Technical Information Center. https://apps.dtic.mil/sti/ citations/AD1039962

Von Bredow, W. (2011). Re-Nationalization of military strategy? New challenges for the Armed Forces in a changing global environment. Military and Society in 21st Century Europe, 45-54. Routledge.

Wade, N. M. (2015). BSS5: The Battle Staff [SMARTbook] (5. ${ }^{\mathrm{a}}$ ed.). The Lightning Press.

Wade N. M. (2017). JFODS5: The Joint Forces Operations \& Doctrine [SMARTbook] (5.a ed.). The Lightning Press.

Yang, M., \& Gabrielsson, P. (2017). Entrepreneurial marketing of international high-tech business-to-business new ventures: A decision-making process perspective. Industrial Marketing Management, 64, 147-160. 